\title{
Long-Distance Quantum Communication With Entangled Photons Using Satellites
}

\author{
Markus Aspelmeyer, Thomas Jennewein, Martin Pfennigbauer, Student Member, IEEE, Walter R. Leeb, and \\ Anton Zeilinger
}

Invited Paper

\begin{abstract}
The use of satellites to distribute entangled photon pairs (and single photons) provides a unique solution for long-distance quantum communication networks. This overcomes the principle limitations of Earth-bound technology, i.e., the range of the order of $100 \mathrm{~km}$ afforded by both optical fiber and by terrestrial free-space links.
\end{abstract}

Index Terms-Quantum communication, quantum entanglement, satellite applications, space technology.

\section{INTRODUCTION}

Q UANTUM entanglement is at the heart of quantum physics [1] and, at the same time, the basis of most quantum communication protocols such as quantum cryptography [2]-[5], quantum dense coding [6], quantum teleportation [7], or methods to exploit the computational advantages of quantum communication complexity [8]-[10]. Each of those schemes allows efficient communication and computation beyond the capabilities of their classical counterparts, which results in a new emerging quantum information technology. This makes a global quantum communication network desirable, where the distribution and manipulation of quantum entanglement on a global scale is a central task. However, while the realization of such schemes is routine work in the laboratory, nontrivial problems emerge in long-distance applications. At present, the only suitable system for long-distance quantum communication are photons. Other systems such as atoms or ions are studied thoroughly, however their

Manuscript received April 25, 2003; revised July 31, 2003. This paper was supported by the Austrian Science Foundation (FWF) and the Alexander von Humboldt Foundation. This paper evolved from a project supported by the European Space Agency under ESTEC/Contract 16358/02/NL/SFe, "Quantum Communications in Space."

M. Aspelmeyer is with the Institut für Experimentalphysik, Universität Wien, A-1090 Wien, Austria (e-mail: aspelm@quantum.at).

T. Jennewein is with the Institut für Quantenoptik und Quanteninformation, Österreichische Akademie der Wissenschaften, A-1090 Wien, Austria (e-mail thomas.jennewein@quantum.at).

M. Pfennigbauer and W. R. Leeb are with the Institut für Nachrichtentechnik und Hochfrequenztechnik, Technische Universität Wien, A-1040 Wien, Austria (e-mail: mpfennig@nt.tuwien.ac.at; Walter.Leeb@tuwien.ac.at).

A. Zeilinger is with the Institut für Experimentalphysik, Universität Wien, A-1090 Wien, Austria and also with the Institut für Quantenoptik und Quanteninformation, Österreichische Akademie der Wissenschaften, A-1090 Wien, Austria (e-mail: anton.zeilinger@quantum.at).

Digital Object Identifier 10.1109/JSTQE.2003.820918 applicability for quantum communication schemes is presently not feasible within the near future, leaving photons as the only choice for long-distance quantum communication. One of the problems of photon-based schemes is the loss of photons in the quantum channel. This limits the bridgeable distance for single photons to the order of $100 \mathrm{~km}$ in present silica fibers [11], [12]. Recent quantum cryptography experiments already achieve such distances [13], [14]. In principle, this drawback can eventually be overcome by subdividing the larger distance to be bridged into smaller sections over which entanglement can be teleported. The subsequent application of so-called "entanglement swapping" [15] may result in transporting of entanglement over long distances. Additionally, to diminish decoherence effects possibly induced by the quantum channel, quantum purification might be applied to eventually implement a full quantum repeater [16]. In fact, the experimental building blocks for a full-scale quantum repeater based on linear optics have been successfully demonstrated over the last years by the realization of teleportation and entanglement swapping [17]-[19] and, only recently, by the implementation of a quantum purification protocol [20]. Two related, recent results, both of relevance for long-distance applications, are the demonstration of quantum state teleportation over a distance of several tens of meters [21] and the first realization of freely propagating teleported quantum bits (qubits) [22], which eventually will allow the subsequent use of teleported states. From the present point of view it seems obvious that a full implementation of a quantum repeater is within reach.

Despite those achievements of quantum communication experiments, the distances over which entanglement can be distributed in a single section, i.e., without a quantum repeater inbetween, are by far not of a global scale. Experiments based on present fiber technology have demonstrated that entangled photon pairs can be separated by distances ranging from several hundreds of meters up to $10 \mathrm{~km} \mathrm{[23]-[25],} \mathrm{but} \mathrm{no} \mathrm{improve-}$ ments by orders of magnitude are to be expected. On the other hand, optical free-space links could provide a unique solution to this problem since they allow in principle for much larger propagation distances of photons due to the low absorption of the atmosphere in certain wavelength ranges. Also, the almost nonbirefringent character of the atmosphere [26] guarantees the preservation of polarization entanglement to a high degree. Free-space optical links have been studied and successfully 
implemented already for several years for their application in quantum cryptography based on faint classical laser pulses [26]-[28]. Recently, we succeeded in demonstrating a next crucial step, namely the distribution of quantum entanglement via a free-space link, which was verified by violating a Bell inequality between two distant receivers without a direct line of sight between them [29].

However, terrestrial free-space links suffer from obstruction of objects in the line of sight, from possible severe attenuation due to weather conditions and aerosols [30] and, eventually, from the Earth's curvature. They are thus limited to rather short distances. To fully exploit the advantages of free-space links, it will be necessary to use space and satellite technology. By transmitting and/or receiving either photons or entangled photon pairs to and/or from a satellite, entanglement can be distributed over truly large distances and thus would allow quantum communication applications on a global scale. Such a scenario looks unrealistic at first sight, but in this paper we will show that the demonstration of quantum communication protocols using satellites is already feasible today. To do so, we will describe possible space scenarios based on entanglement. We then analyze prerequisites to distribute entanglement via satellites, describe experimental scenarios for first proof-of-principle experiments and finally give an outlook on the perspectives of satellite-aided quantum communication.

\section{SCENARIOS OF SPACE EXPERIMENTS}

When considering space scenarios that allow the distribution of entangled photon pairs, we can distinguish the cases in which a satellite is used to carry either: i) a transmitter of entangled photons, or ii) a receiver, or iii) a relay station to distribute photons to further locations. These scenarios will permit different applications.

\section{A. Earth-Based Transmitter Terminal}

The scenarios involving an Earth-based transmitter terminal allow to share quantum entanglement either between ground and satellite, or between two ground stations, or between two satellites and thus to communicate between such terminals employing quantum communication protocols. In the most simple case, a straight uplink to one satellite-based receiver [see Fig. 1(a)] can be used to perform secure quantum key distribution (QKD) between the transmitter station and the receiver. Here, one of the photons of the entangled pair is being detected right at the transmitter site and thus the entangled photon source is used as a triggered source for single photons. If the satellite acts as a relay station [see Fig. 1(b)], the same protocol can be established between two distant Earth-based communication parties. Shared entanglement between two parties can be achieved by pointing each of the photons of an entangled pair either toward an Earth-based station and a satellite or toward two separate satellites [see Fig. 1(c) and (d)]. Another set of satellite-based relays can be used to further distribute the entangled photons to two ground stations [see Fig. 1(e)]. Possible applications for shared entanglement between two parties are QKD or entanglement-enhanced communication protocols [31].
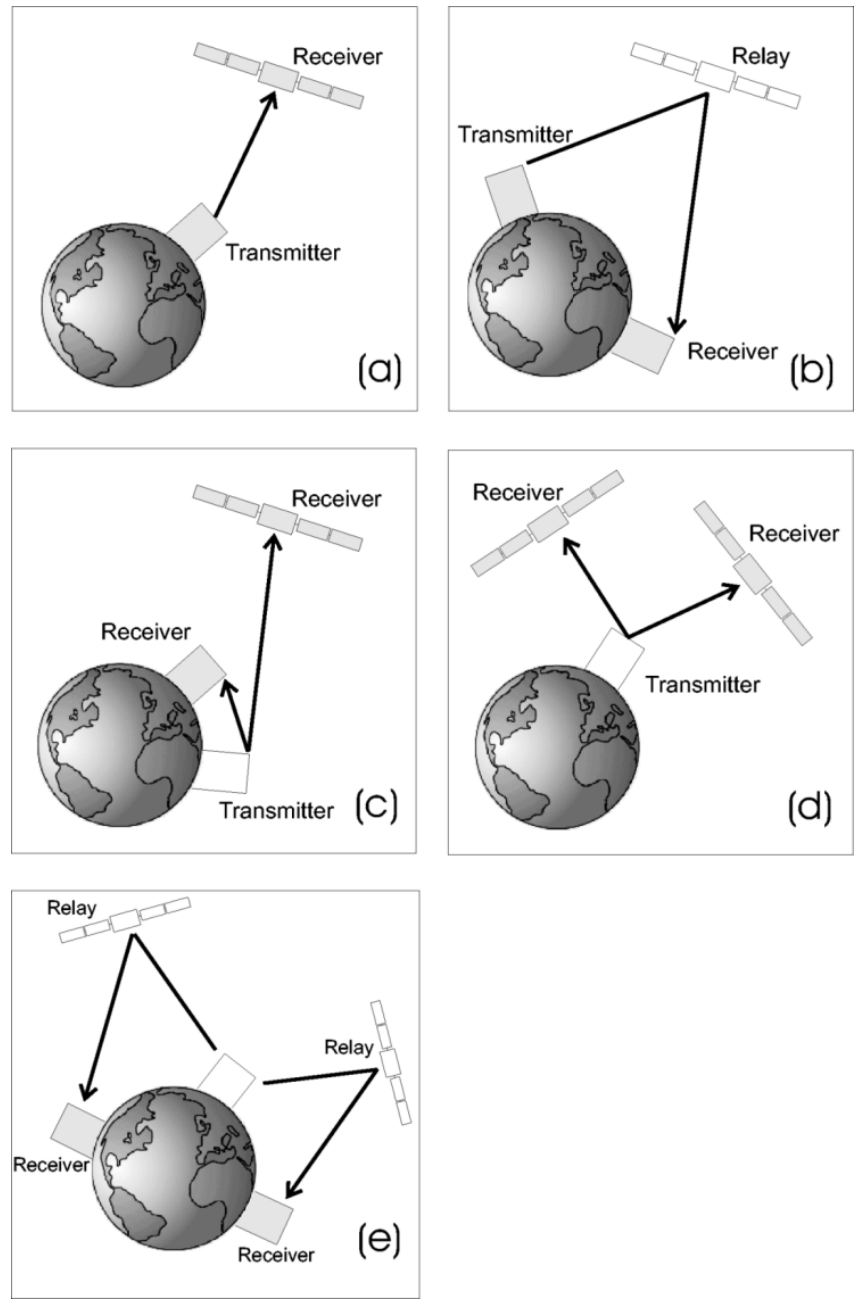

Fig. 1. Scenarios for satellite-aided quantum communication with an Earth-based transmitter terminal. The transmitter terminal distributes entangled photon pairs to the receivers which can perform an entanglement-based quantum communication protocol. As indicated, a relay module redirects and/or manipulates qubit states without actually detecting them.

\section{B. Space-Based Transmitter Terminal}

In a second scenario, a transmitter with an entangled photon source is placed on a space-based platform. This allows not only longer link distances because of reduced influence of atmospheric turbulence (see Appendix II). It will also be the preferred configuration for global quantum communication, since only one downlink per photon of the entangled pair is necessary to share entanglement between two Earth-based receivers. Again, already a simple downlink allows one to establish a single-photon link, e.g., for quantum cryptography [see Fig. 2(a)]. In this configuration, a key exchange between two ground stations is also possible. To this end each of the two ground stations has to establish a quantum key with the satellite. Since the space terminal has access to both keys, it can transmit a logical combination of the keys, which can then be used by either ground station or both ground stations such that they arrive at the same key. This logical combination can easily be chosen in a way that it cannot reveal any information about the key. Note that the key does not have to be generated simultaneously at both receiver stations. In principle, a quantum key exchange can be performed between arbitrarily located 

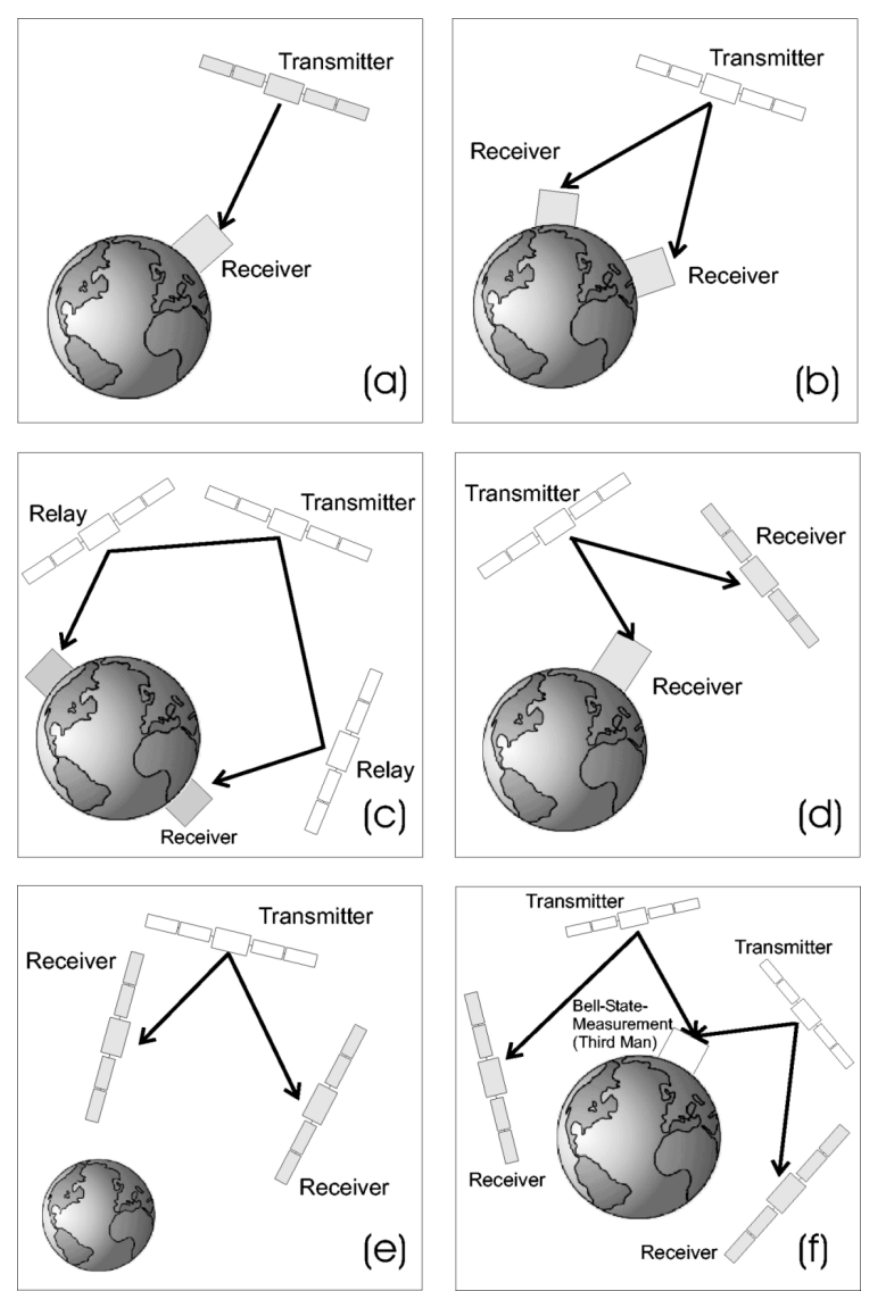

Fig. 2. Scenarios for quantum communication with a space-based transmitter terminal. Since optical space-to-Earth downlinks are less effected by atmospheric turbulence such configurations provide lower overall link attenuations and thus allow longer distances than the corresponding Earth-to-space links.

ground stations. This is also possible for a ground-based transmitter terminal as shown in Fig. 1(a). However, in all such scenarios based on single photons the security requirement for the transmitter terminal is as high as it is for the ground station. Only the use of entangled states sent to two separate ground stations allows instantaneous key exchange between these two communicating Earth-bound parties and also relaxes the security requirement for the transmitter module. Furthermore, more advanced quantum communication schemes will be feasible. The required shared entanglement can be established either by two direct downlinks [Fig. 2(b)] or by using additional satellite relay stations [Fig. 2(c)]. Quantum entanglement can also be distributed between a ground station and a satellite [Fig. 2(d)] or between two satellites [Fig. 2(e)]. In an even more elaborate scheme a third party, either on ground or on a satellite, might be involved, which is capable of performing a Bell-state analysis on two independent photons [Fig. 2(f)]. This allows quantum state teleportation and even entanglement swapping and could thus resemble a large-scale quantum repeater for a truly global quantum communication network. Applied to quantum cryptography, this third party might be used to control the communication between the two other parties in a "Third Man" cryptography protocol. For example, in a polarization-based experiment, depending on whether he performs a simple polarization analysis of the independent photons or a Bell-state measurement, the "Third-Man" can communicate secretly with either of the two parties (or with both) or he can control whether the two can communicate secretly or not without knowing the content of the communication.

\section{Link Requirements}

The maximal acceptable link attenuation for a quantum communication system based on entangled photons is determined by the timing resolution and the dark count rates of the detectors used, as well as by the net production rate of the source. As the minimum signal-to-noise ratio (SNR), we assume the one that it is necessary for the violation of a Bell-inequality because of its relevance to establish the security of quantum cryptography (see Appendix I). With a typical overall detection efficiency $\eta_{\text {Det }}=0.3$, a photon production rate of $P=5 \cdot 10^{5} \mathrm{~s}^{-1}$, an estimated total background count rate ${ }^{1}$ of $S=10^{3} s^{-1}$ and a coincidence timing window of $\Delta \tau=5 \cdot 10^{-9} \mathrm{~s}$, the link efficiency should obey

$$
\eta_{\text {link }} \geq 6.66 \ldots \cdot 10^{-7}
$$

when following the calculations presented in Appendix I. Roughly speaking, a total link efficiency of $\eta_{\text {link }}=\eta_{\text {link } 1} \eta_{\text {link2 }} \approx 10^{-6}(-60 \mathrm{~dB})$ is necessary.

The link attenuation is also important for determining the number of photon pairs that can be received within a certain time window. This could be crucial in scenarios where the links are only available for short times as is for example the case in uplinks to low orbiting LEO satellites.

\section{LINK ATTENUATION}

The overall link efficiency of $10^{-6}$, corresponding to a maximum attenuation of $60 \mathrm{~dB}$, imposes quite a strong restriction on the various space scenarios. In the following, we will investigate the link attenuation for optical free-space links involving space infrastructure. The attenuation factor calculated includes the effect of beam diffraction, of attenuation and turbulence-induced beam spreading caused by the atmosphere, of receive aperture diameter, of losses within the telescopes acting as antennas, as well as antenna pointing loss. Effects not included in this factor are the detection efficiency of the photon-counter modules ( $\sim 3.5 \mathrm{~dB}$ per detector) and reflective and absorptive losses at optical components (typically $\sim 3 \mathrm{~dB}$ per individual photon link), which add up to an overall detection efficiency of $\sim 6.5 \mathrm{~dB}$ per photon link. One may take these losses into account when calculating specific total link budgets. Fig. 3 summarizes the scenarios considered based on satellites in geostationary orbit (GEO) and in low earth orbit (LEO). Such satellites may serve

\footnotetext{
${ }^{1}$ This includes the dark counts from the detector $\left(50 \mathrm{~s}^{-1}\right)$ and additional background contributions from sunlight eventually reflected by the ISS $\left(950 \mathrm{~s}^{-1}\right)$. Our estimate is based on an $0.1 \mathrm{~nm}$ bandwidth spectral filtering at the receiver site and on a sufficiently narrow field of view of the receiver telescope [32]. To avoid the significantly higher background radiation from the sky during the day the assumed background rate can only be achieved at nighttime.
} 


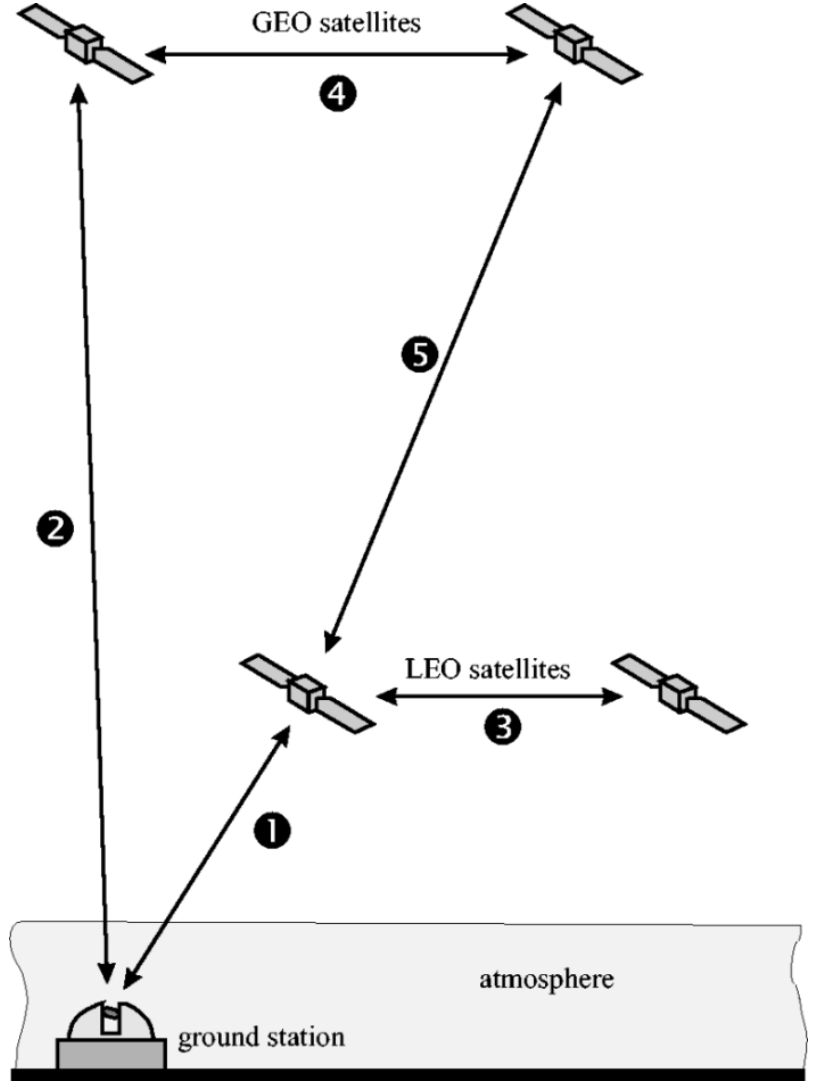

Fig. 3. Summary of links involving Earth-based ground stations and LEO satellites or GEO satellites.

as a platform for transmitters or receivers. We presently do not envision the use of passive relays, e.g., retroreflectors or mirrors, because of the high link loss they would introduce and because of the difficulty to implement a point-ahead angle. ${ }^{2}$

\section{A. Satellite-Ground Links}

1) Ground-LEO or LEO-Ground Links: For the case of a LEO-based transmitter or receiver (Link 1 in Fig. 3), link attenuation poses no problems. Even for quite small telescopes onboard the LEO satellite, the attenuation factor is well below $60 \mathrm{~dB}$ for all cases. Fig. 4 is a contour plot of the link attenuation as a function of transmitter and receiver aperture diameter $\left(D_{T}\right.$, $D_{R}$ ) for the ground-to-LEO uplinks operated at a wavelength of $\lambda=800 \mathrm{~nm}$. Two additional vertical scales give the link distance $\mathrm{L}$ for $30 \mathrm{~cm}$ receive telescope aperture as well as for the receive telescope aperture for a link distance of $\mathrm{L}=500 \mathrm{~km}$ (For the equation and further parameters used to arrive at Fig. 4-and also at some subsequent figures—see Appendix II.). The lines of equal attenuation are separated by $5 \mathrm{~dB}$. The corresponding plot for LEO-to-ground downlinks is shown in Fig. 5. One notes that the attenuation is much larger for the uplink than for the downlink. This is caused by the pronounced influence of atmospheric turbulence for the uplink, where the turbulent layers are close to the transmitter. In contrast, for a downlink the effect of the

\footnotetext{
${ }^{2}$ The point-ahead angle denotes the difference angle between transmit and receive direction of the telescope. Its occurrence is a consequence of the movement of the satellite together with the finite velocity of light of the signal propagation.
}

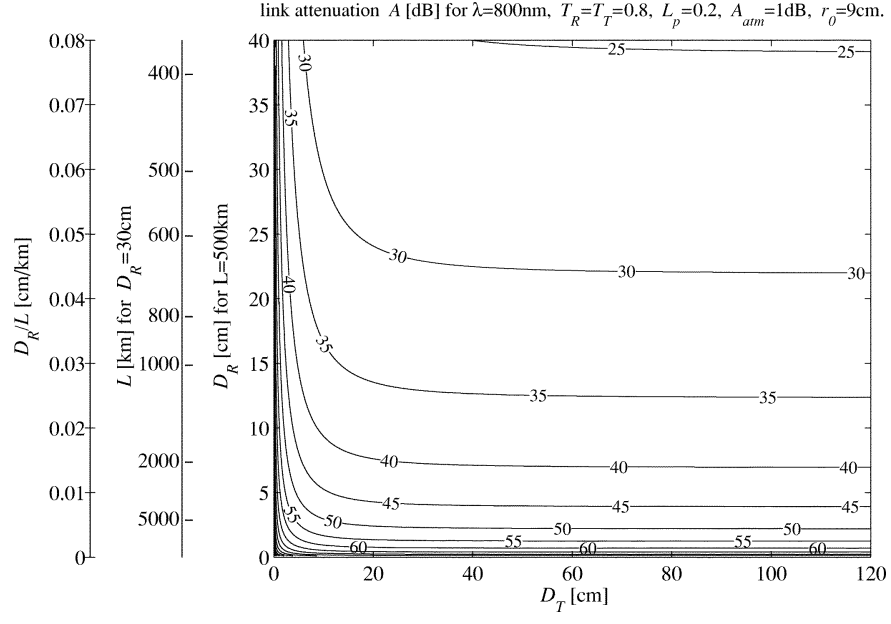

Fig. 4. Contour plot of link attenuation $A$ (in $\mathrm{dB}$ ) as a function of transmitter and receiver aperture diameter $\left(D_{T}, D_{R}\right)$ and link distance $L$ for ground-to-LEO uplinks at $\lambda=800 \mathrm{~nm}$.

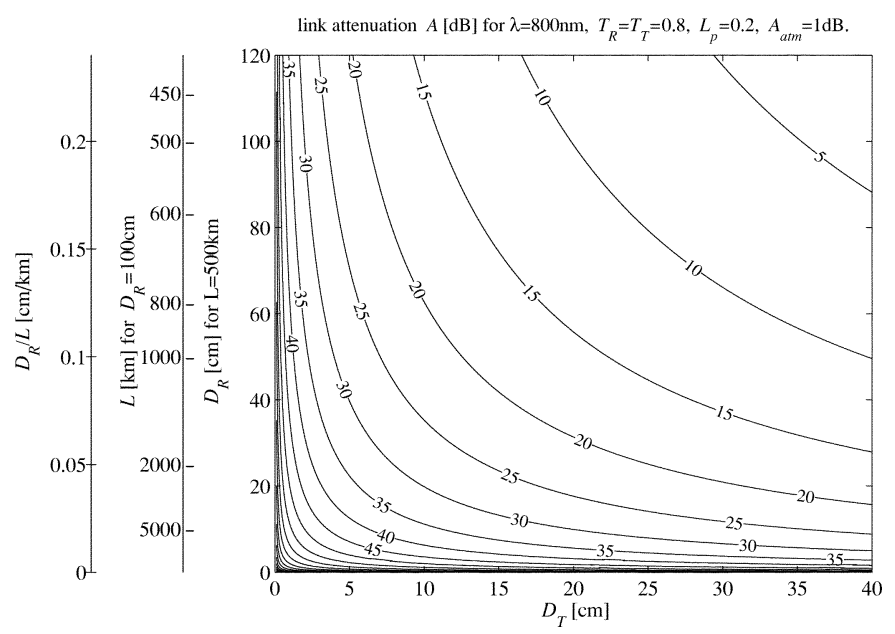

Fig. 5. As Fig. 4 but for LEO-to-ground downlinks.

turbulent layer close to the receiver is negligible to first order. Another consequence of turbulence is that increasing the transmitter aperture for the uplink beyond $40 \mathrm{~cm}$ hardly decreases the link attenuation (see Fig. 4). This effect could be migitated by employing adaptive optics.

In the case of links connecting a LEO and a ground station, the possible duration for communication is comparatively short (e.g., a few minutes) and the angular velocity with which the telescope at the ground station has to be moved to track the satellite along its orbit is high. For all ground-to-space links the possibility of communication is weather dependent. While for clear weather and sufficient altitude of the ground station the uplink attenuation caused by the atmosphere is mainly determined by turbulence-induced beam spread, ${ }^{3}$ clouded skies will make any link impossible. This influence increases at low elevation angles typical for LEO-to-ground links. This lengthens the propagation path within the atmosphere.

2) Ground-GEO or GEO-Ground Links: The long distance in links between GEO and ground (Link 2 in Fig. 3) results in

\footnotetext{
${ }^{3}$ For a diffraction limited telescope of $20 \mathrm{~cm}$ diameter and a link distance of $500 \mathrm{~km}$, some $20 \mathrm{~dB}$ attenuation have to be expected [33]
} 
TABLE I

LiNK ATTENUATION FOR VARIOUS SPACE SCENARIOS

\begin{tabular}{c||c|c|c}
\hline $800 \mathrm{~nm}$ & $\begin{array}{c}\text { ground-based } \\
\text { receiver }\end{array}$ & $\begin{array}{c}\text { LEO } \\
\text { receiver }\end{array}$ & $\begin{array}{c}\text { GEO } \\
\text { receiver }\end{array}$ \\
\hline \hline $\begin{array}{c}\text { ground } \\
\text { based } \\
\text { transmitter }\end{array}$ & & $27.4 \mathrm{~dB}$ & $64.5 \mathrm{~dB}$ \\
\hline $\begin{array}{c}\text { LEO } \\
\text { transmitter }\end{array}$ & $6.4 \mathrm{~dB}$ & $28.5 \mathrm{~dB}$ & $52.9 \mathrm{~dB}$ \\
\hline $\begin{array}{c}\text { GEO } \\
\text { transmitter }\end{array}$ & $43.6 \mathrm{~dB}$ & $52.9 \mathrm{~dB}$ & $53.9 \mathrm{~dB}$ \\
\hline
\end{tabular}

For the numerical calculation the default parameter values given in Appendix III have been taken at a wavelength of $800 \mathrm{~nm}$.

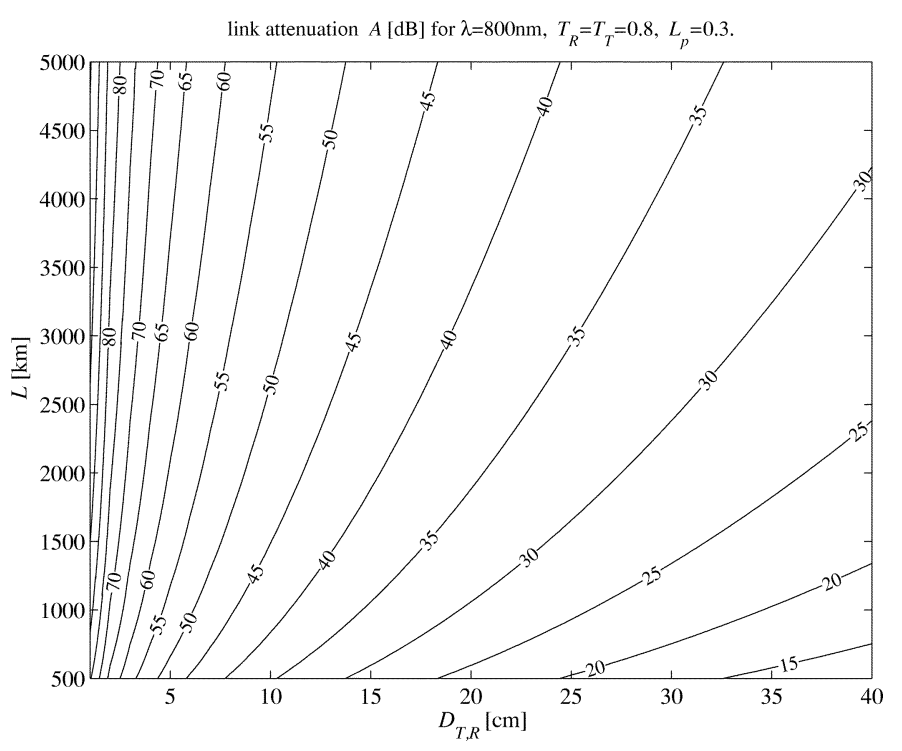

Fig. 6. As Fig. 4 but for LEO-to-LEO links.

a relatively high attenuation. As a consequence, with a ground station aperture of $D=100 \mathrm{~cm}$ and a GEO terminal aperture of $D=30 \mathrm{~cm}$ one will be able to meet the $60 \mathrm{~dB}$ requirement in a downlink, but not in an uplink (compare Table I of Section V).

\section{B. Satellite-Satellite Links}

While from a technological point of view a satellite-to-satellite link is the most demanding configuration it offers highly attractive prospects for scientific experiments. It allows one to cover, in principle, arbitrarily large distances and might thus also be a possibility for further novel fundamental tests on quantum entanglement.

We calculated the attenuation factor as a function of both transmitter and receiver aperture diameter for a LEO-LEO link (Link 3 in Fig. 3). Fig. 6 displays the attenuation for a wavelength of $800 \mathrm{~nm}$ as a function of the satellite distance $L$ and telescope diameters $D_{T}$ and $D_{R}$, assumed to be equal for both terminals. We conclude, that the $60-\mathrm{dB}$ limit poses no problem for LEO-LEO links with reasonable link distance.

For GEO-GEO links (Link 4 in Fig. 3), the attenuation can be read off Fig. 7, where again equal telescope apertures have been assumed. For a distance of $L=45000 \mathrm{~km}$, an attenuation of $A=55 \mathrm{~dB}$ would result for $D_{T}=D_{R}=30 \mathrm{~cm}$.

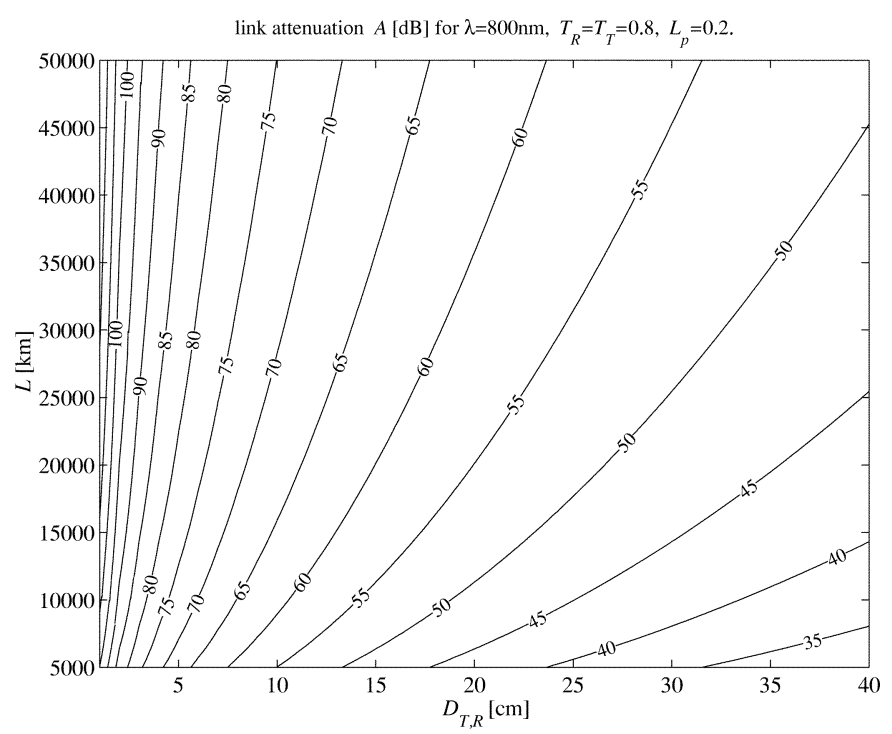

Fig. 7. As Fig. 4, but for GEO-to-GEO links.

\section{TeChNological PreRequisites for ENTANGLEMENT IN SPACE}

For performing the quantum communication experiments aforementioned, the following minimum hardware is required: a transmitter terminal to generate and transmit the entangled particles and one or more receiver terminals suited for single-photon manipulation and detection. We briefly outline their main features.

\section{A. Transmitter, Receiver, and Relay Modules}

A transmitter module comprises a photon source for entangled photon pairs (including passive or active manipulation of single qubit-states), a module for timing synchronization with the receiver station and a channel for classical communication. Present entangled photon sources rely on laser-pumped spontaneous parametric down conversion [34]. This technology is very likely capable of being miniaturized to a size suitable for satellite modules. Elsewhere, we have demonstrated a compact and stable source for entangled photon pairs that could be operated outside the shielded laboratory environment [29].

A receiver module comprises one ore more optical input channels, each of which allows independent manipulation of qubits such as the rotation of photon polarization or the modulation of an interferometric phase. Additionally, it has to be equipped with single-photon detectors at each input port, a receiver module for timing-synchronization, and a classical channel for communication with the transmitter. Depending on whether active (remote) control of optical elements for qubit manipulation is possible or not (via, e.g., a polarizer or a retarder), we distinguish between active and passive receiver modules. Passive manipulation only requires a static setup of linear optics components. Typically, beam splitters in the input ports would randomly distribute incoming photons to differently oriented retarders, polarizers or beam splitters, where a manipulation and successive detection of single photons takes place. This kind of passive receiver module has 
recently also been suggested by Rarity et al. in a space-suited system for single-photon QKD [35]. For active control of single qubit manipulation, an additional information concerning the arrival time (i.e., a timing synchronization) is required. More advanced quantum communication schemes, such as quantum dense coding or quantum teleportation, require at some stage of the protocol the projection of independent photons in a joint Bell state, i.e., a Bell-state measurement. Up to now, efficient achievement of this projection is only possible by means of two-particle-interferometry [6]. ${ }^{4}$ This requires that the arrival time difference of two photons at the two input ports of a beam splitter has to be less than their coherence time (typically $0.5 \mathrm{ps}$ ) [37].

A relay module would redirect and/or manipulate qubit states without actually detecting them. Possibilities for its implementation range from a simple retroreflector to a more sophisticated relay-satellite (e.g., for deep-space communications), where the entanglement of photons establishing the quantum channel between ground station and deep-space satellite could be purified. We emphasize, that a relay can not serve as an amplifier. This is a consequence of the quantum no-cloning theorem [38].

\section{B. Existing Optical Space Technology}

Optical transceivers for space-to-ground links or intersatellite links are almost state of the art. The major design parameters for the transmission subsystem are laser wavelength, modulation format, data rate, and reception technique. Of equal importance is the subsystem required for beam pointing, link acquisition, and automatic mutual terminal tracking (PAT). Because of the very narrow widths of the communication beams involved, PAT asks for highly sophisticated protocols and for electromechanic and electrooptic hardware meeting exceptional technological standards. Major parameters entering the link capacity are telescope size, optical transmit power, link distance, and receiver sensitivity. Other aspects are mass, volume, and power consumption of the terminal. Examples for existing space laser communication links include ESA's intersatellite link Semiconductor Laser Intersatellite Link Experiment (SILEX) and a satellite ground link, which was only recently realized between the GEO satellite ARTEMIS and ESA's optical ground station OGS at Tenerife.

Photon sources and detectors presently implemented in such classical space laser communication systems can, in general, not be directly employed in quantum communication systems. However, the experience available may serve as a starting point for the development of space qualified components needed for quantum space experiments. The available optical communication technology could of course be applied to provide the classical channel that is always necessary in parallel to the quantum channel. One would also make synergistic use of some of the optics employed for PAT and employ one and the same telescope as antenna for both the classical and the quantum channel, which is a novel way of quantum-classical-multiplexing.

\footnotetext{
${ }^{4}$ Recently, another scheme based on the nonlinear couplings has been suggested to achieve a full discrimination of all four Bell states although with low efficiency [36].
}

\section{Proof-of-Principle Experiments}

The establishment of entanglement in space and, subsequently, its use for fundamental quantum physics experiments and quantum communication applications necessitates certain experimental stages:

Stage I: Creation and detection of qubits (here: single photons) via an optical space link. From an application point of view this achievement would already allow to perform QKD based on single photons.

Stage II: Establishment of entanglement (i.e. nonclassical correlations via shared entangled particles) between the communicating parties. This includes the ability to detect single qubits synchronously at the spatially separated locations of the communicating parties. At this stage already the most fundamental experiment in quantum physics, demonstration of a violation of Bell's inequality can be performed. It also makes possible further experiments such as QKD based on entangled qubits.

Stage III: Bell-state analysis of independent qubits. For the case of photons, the most efficient scheme relies on two-photon interferometry at a beam splitter. Technically speaking, the arrival time of the photonic qubits at the receiver module has to be synchronized such that photon wavepackets overlap at the beam splitter within their coherence length. If this problem is solved, all advanced quantum communication and computation protocols such as quantum-state teleportation or quantum dense coding can be implemented.

The selection of an experimental scenario, in which all of these stages can be performed, requires a tradeoff between link attenuation and experimental flexibility. It has been shown above that the total link attenuation of the experimental setup must not exceed some $60 \mathrm{~dB}$, assuming today's quantum-optics technology. Presently, without the use of quantum memories, entanglement can only be shared when more than one link is available. For the symmetric case of two equally long quantum links (one transmitter and two receivers), this limits the maximal single-link attenuation (between one transmitter and one receiver) to approx. $30 \mathrm{~dB}$. Although space-to-space links have the attractive advantage of not being influenced by the Earth's atmosphere, we have to discard them at present due to the expected disproportionate technological and financial effort as compared to alternative schemes with at least one of the communication terminals on ground. Since two links should be established, it is therefore most reasonable to place the transmitter module in space, while the receiver modules stay in easily accessible ground-based laboratories. Most envisioned quantum experiments require higher flexibility at the receiver due to active polarization control or data analysis. Also, the atmosphere causes a larger footprint in an uplink than in a downlink, due to the higher influence of turbulence. With such perspectives it becomes obvious that in a first proof-of-principle experiment one should place the transmitter module into space and the receiver module(s) on Earth. Because of their relative stationarity, terminals placed on GEO satellites do not require such a highly sophisticated pointing, acquisition and tracking (PAT) systems as those on a LEO satellite. They would also 
allow for long-duration experiments. On the other hand, the link attenuation and cost are significantly larger for GEO links compared to LEO links. Therefore, when trading GEO-based against LEO-based systems, we would rather accept the more complex PAT system and the limited connection time per orbit and suggest to use a LEO platform for the transmitter terminal for first proof-of-principle experiments.

\section{Perspectives and Limits of Space-Aided QUANTUM COMMUNICATIONS}

We determined the typical attenuation for the links indicated in Fig. 3. The values listed in Table I were calculated using (7) for wavelengths of $800 \mathrm{~nm}$, assuming telescope apertures characteristic for present day optical space technology. Furthermore, calculations have been performed for a wavelength of $1550 \mathrm{~nm}$. The first wavelength seems reasonable because the best singlephoton detectors exist for $800 \mathrm{~nm}$, while the second wavelength is mainly used in standard telecom systems. Applying this wavelength may thus increase compatibility and reduce development effort because commercial off-the-shelf components are available. However, the total link attenuation is slightly increased for $\lambda=1550 \mathrm{~nm}$ due to the higher beam divergence at larger wavelength, although the atmospheric absorption and the influence of turbulent air is slightly decreased compared to $800 \mathrm{~nm}$. For each link the default parameters are specified in Appendix III.

Based on present-day technology and assuming reasonable link parameters, it seems feasible to achieve enough entangled photons per receiver pair to demonstrate a quantum communication protocol. For example, assuming a LEO-based transmitter terminal, a simultaneous link to two separate receiving ground stations and a (conservatively estimated) total link attenuation of approx. $51 \mathrm{~dB},{ }^{5}$ one can expect a local count rate of approximately 2600 per second in total at each of the receiver terminals. The number of shared entangled photon pairs is then expected to be approximately 4 per second. For a link duration of $300 \mathrm{~s}$, this accumulates to a net reception of 1200 entangled qubits. One can expect erroneous detection events on the order of 7 per 100 $\mathrm{s}$, which yields a bit error of approximately $2 \%$. This would already allow a QKD protocol between the two receiver stations. It is thus clear, that a demonstration of basic quantum communication protocols based on quantum entanglement can already be achieved today.

All proposed setups are based on the utilization of entangled photon pairs as carriers of quantum information. Given state-of-the-art technologies present in today's quantum optics labs we can specify some practical limitations for the preparation and detection of entangled qubits. ${ }^{6}$

The rate of information transfer is limited by the maximal number of photons or entangled photon pairs that can be created and detected. Typical standard repetition rates for pulsed laser

\footnotetext{
${ }^{5}$ Note again, that without quantum memories, quantum entanglement can only be shared when more than one link is available. For the symmetric case, i.e., two equally long quantum links involving one transmitter and two receivers, this limits the maximal single link attenuation between one transmitter and one receiver to approximately $30 \mathrm{~dB}$. Here, we assume a loss of $25.5 \mathrm{~dB}$ for each of the downlinks.

${ }^{6} \mathrm{~A}$ more detailed analysis on link duration and qubit transmission rates for specific experimental scenarios is presented in [39].
}

sources able to create (entangled) qubit states are of the order of $10^{6}-10^{7} \mathrm{~s}^{-1}$, which will be reduced to only a few thousands per second by effects such as optical filtering, finite coupling efficiency and finite detection efficiency. Additionally, one has to take into account transmission losses, which can limit the qubit transmission drastically. Also, state of the art detector systems have low dynamic ranges over a maximum of six orders of magnitude $^{7}$ and a maximal detection rate of some MHz. ${ }^{8}$ Further development both of source and detector technology will lead to additional improvements of the qubit rates.

In the present paper, we propose various scenarios for entanglement-assisted global and space-based quantum communication. While this in itself is already an interesting and outstanding experimental challenge, there are various specific novel opportunities which such a technology would offer. First and from a technological perspective foremost is the possibility to establish a secure and efficient communication using the methods of entanglement based quantum cryptography. As we explained above, that way one could not only establish a secure communication link between two arbitrary locations on Earth. It would also be possible to establish secure communication from Earth to satellites or between satellites. This would certainly increase the security of satellite remote control, which at present is a highly sensitive area and, thus far, an unsolved technical problem.

Another very important aspect is the fact that resources for communication in space are usually very limited, rare and expensive, particularly in view of the necessity of low power consumption. There we expect that quantum communication provides a natural goal, where one utilizes the ultimate limit of transmitting one bit of information per photon. Actually, this could even be increased if one would exploit photon states in higher dimensional Hilbert-spaces, where one photon can carry more information than one bit [40]. This might be particularly useful in deep space communication, where both communication time and energy resources are even more limited and where efficient communication is absolutely mandatory. One might conceive that the ultimate application of novel ideas in quantum communication complexity might some day provide a unique means to reduce the resources necessary for the transmittal of certain information. Finally, we mention that the distribution of quantum entanglement over astronomic scales will allow the test of quantum mechanics, particularly via violation of Bell's Theorem over novel distances larger by several orders of magnitude than existing experiments. This will make the issues of quantum nonlocality even more directly obvious than today. One might even conceive that in future manned missions to other planets, say to Mars, one could actually have human observers who decide to perform these experiments in the sense that the decision, which parameter is measured is made by human observers in the last instance when the entangled photons are already on their way. This would eliminate any hidden possibility of crypto-determinism, except the last resource namely that even human decisions are deterministic. Furthermore, as it has always been the

\footnotetext{
${ }^{7}$ The highest dynamic range of about six orders of magnitude is achieved with $\mathrm{Si}$ avalanche photodiodes (APDs), whereas systems based on InGaAs up to now reach only three to four orders of magnitude.

${ }^{8}$ Some tens of $\mathrm{kHz}$ in the case of InGaAs.
} 
case in physics, we expect that novel ideas will emerge as soon as such systems have been placed in space and are safely operating.

\section{APPENDIX I}

\section{LINK REQUIREMENTS}

The accidental coincidence rate is given by

$$
C_{\text {acc }}=S_{1} S_{2} \Delta \tau
$$

where $S_{1}, S_{2}$ are the background count rates of the two detectors and $\Delta \tau$ is the timing resolution for the electronic registration of a twofold coincidence event. As the minimum SNR, we assume that required for violating a Bell-inequality, since this guarantees at the same time the security of certain quantum cryptography schemes [41]. For the case of polarization-entangled photons this necessitates a twofold coincidence visibility of at least $71 \%$, corresponding to a SNR of 6:1.9 Below that ratio a local realistic modeling of the observed correlations is possible thus allowing unobserved eavesdropping. ${ }^{10}$ Therefore, in order to discriminate the signal from the background coincidences, the coincidence rate $C_{0}$ must be at least six times larger than $C_{\text {acc }}$.

The coincidence detection rate is determined by the total coincidence efficiency $\eta_{\text {link }}$, which is the product of the individual efficiencies for the two qubit links

$$
\eta_{\text {link }}=\eta_{\text {Link } 1} \eta_{\text {Link2 }}
$$

The detected signal coincidences $C$ are given by the product

$$
C=P \eta_{\text {link }} \eta_{\operatorname{det} 1} \eta_{\operatorname{det} 2}
$$

where $P$ is the pair production rate of the source and $\eta_{\operatorname{det} 1}, \eta_{\operatorname{det} 2}$ are the detection probabilities. In order to achieve a violation of Bell's inequality, the signal coincidences must exceed the limit $C_{0}=S N R \cdot C_{\text {acc }}$, which leads to the following limit for the total link efficiency

$$
\eta_{\text {link }} \geq S N R \frac{C_{\mathrm{acc}}}{P \eta_{\operatorname{det} 1} \eta_{\operatorname{det} 2}}=S N R \frac{S_{1} S_{2} \Delta \tau}{P \eta_{\operatorname{det} 1} \eta_{\operatorname{det} 2}} .
$$

\section{APPENDIX II}

\section{MODELING THE LINK ATTENUATION}

We define the link attenuation factor $A$ as the ratio of the mean transmit and receive power $P_{T}$ and $P_{R}$ [43] measured at the entrance and the exit of the transmit and the receive telescope, respectively. Thus losses due to single photon detection efficiency and optical elements such as filters, polarizers or retarders are not included in this number. Then the attenuation factor $A$ of a one-way free-space link is thus given by

$$
A=\frac{L^{2} \lambda^{2}}{D_{T}^{2} D_{R}^{2}} \frac{1}{T_{T}\left(1-L_{P}\right) T_{R}}
$$

where $L$ is the link distance, $\lambda$ the wavelength, and $D_{T}$ and $D_{R}$ the diameters of the transmit and receive telescope. With $T_{T}$ and

\footnotetext{
${ }^{9}$ The visibility, in terms of the signal $S$ and the noise $N$, is defined by $(S-$ $N) /(S+N)$.

${ }^{10}$ Note, that phase-coded entanglement results in slightly higher requirements to show that no local realistic model describes the corresponding correlations [42].
}

$T_{R}$ we denote the transmission factors $(\leq 1)$ of the telescopes, $L_{P}$ is the pointing loss due misalignment of transmitter and receiver. This basic relationship applies (i) if the receiver is in the transmitter's far field, i.e. $L \geq D_{T}^{2} / \lambda$, (ii) if the transmit telescope is diffraction limited, and (iii) if there is no influence of the atmosphere.

\section{Influence of Atmosphere}

Atmospheric effects on propagation at optical beams can be divided into three categories: absorption, scattering, and turbulence [44], [45]. While absorption and scattering mainly depend on wavelength and visibility conditions, the net impact of atmospheric turbulence additionally depends on elevation angle and direction of transmission [46]. The main effect of atmospheric turbulence is an enlarged beam divergence, resulting in a reduced amount of signal power collected by the receive telescope. Further turbulence-induced effects are beam-wander, loss of coherence, scintillation and pulse distortion and broadening ${ }^{11}$ [47]. The effect of turbulence is in general quite different for a space-to-ground link and a ground-to-space link. In a space-to-ground link the light propagates through vacuum for the most of the distance first before being disturbed by the atmosphere, whereas for a ground-to-space link the beam spreading effects of turbulence take place at the beginning of the propagation, causing a strongly enhanced divergence.

\section{Ground-to-Space Links}

For ground-to-space links we therefore modify (6) to take into account an additional attenuation by the atmosphere and the influence of turbulence. The diffraction-limited divergence caused by the aperture diameter of the transmit telescope is increased when the beam passes the turbulent atmosphere. The influence of the atmosphere can be taken into account by the so-called Fried parameter, $r_{0}$, which can be interpreted as an "effective aperture" [46]. We will assume that the divergence due to turbulence adds quadratically to the divergence of the telescope [33]. The attenuation factor may then be approximated by

$$
A=\frac{L^{2}\left(\theta_{T}^{2}+\theta_{a t m}^{2}\right)}{D_{R}^{2}} \frac{1}{T_{T}\left(1-L_{P}\right) T_{R}} 10^{\frac{A_{a t m}}{10}}
$$

where $A_{a t m}$ is the attenuation of the atmosphere, given in $\mathrm{dB}$. The divergence angle resulting from the transmit telescope is assumed to be

$$
\theta_{T}=\frac{\lambda}{D_{T}}
$$

and the turbulence causes the additional divergence

$$
\theta_{\text {atm }}=\frac{\lambda}{r_{0}} \text {. }
$$

This calculation probably underestimates the turbulence effect, ${ }^{12}$, but our model is considered to be suitable to calculate a lower-bound estimation for the attenuation factor.

\footnotetext{
${ }^{11}$ Turbulence-induced pulse distortion and broadening might actually impose an upper limit to the spectral bandwidth in the pulsed downconversion schemes.

${ }^{12}$ In comparison to the values presented in [48] the divergence obtained with our model is lower by a factor of 1.5 . However, we do not know the exact turbulence conditions assumed in [48]. Also, the experimental results of the ARTEMIS-OGS downlink are slightly worse than our calculations would predict.
} 
TABLE II

PARAMETERS FOR GROUND-LEO AND LEO-GROUND LINKS (DEFAULT VALUES ARE UNDERLINED)

\begin{tabular}{l|c|l}
\hline link distance & $L$ & $\underline{500}$ to $1400 \mathrm{~km}$ \\
\hline ground aperture & $D_{T}, D_{R}$ & $1 \mathrm{~m}$ \\
\hline LEO aperture & $D_{T}, D_{R}$ & 20 to $30 \mathrm{~cm}$ \\
\hline wavelength & $\lambda$ & $800 \mathrm{~nm}, 1550 \mathrm{~nm}$ \\
\hline telescope transmission factor & $T_{T}=T_{R}$ & 0.8 \\
\hline pointing loss & $L_{p}$ & 0.2 \\
\hline atmospheric attenuation & $A_{a t m}$ & $1 \mathrm{~dB}$ \\
\hline Fried parameter & $r_{0}$ & $9 \mathrm{~cm}, 20 \mathrm{~cm}$ \\
\hline
\end{tabular}

TABLE III

PARAMETERS FOR GROUND—GEO AND GEO—GROUND LINKS

\begin{tabular}{l|c|l}
\hline link distance & $L$ & $\geq 36000 \mathrm{~km}$ \\
\hline ground aperture & $D_{T}, D_{R}$ & $1 \mathrm{~m}$ \\
\hline LEO aperture & $D_{T}, D_{R}$ & 20 to $30 \mathrm{~cm}$ \\
\hline wavelength & $\lambda$ & $800 \mathrm{~nm}, 1550 \mathrm{~nm}$ \\
\hline telescope transmission factor & $T_{T}=T_{R}$ & 0.8 \\
\hline pointing loss & $L_{p}$ & 0.2 \\
\hline atmospheric attenuation & $A_{a t m}$ & $1 \mathrm{~dB}$ \\
\hline Fried parameter & $r_{0}$ & $9 \mathrm{~cm}, 20 \mathrm{~cm}$ \\
\hline
\end{tabular}

TABLE IV

PARAMETERS FOR LEO-LEO LINKS

\begin{tabular}{l|c|l}
\hline link distance & $L$ & $2000 \mathrm{~km}$ \\
\hline aperture & $D_{T}=D_{R}$ & 20 to $30 \mathrm{~cm}$ \\
\hline wavelength & $\lambda$ & $800 \mathrm{~nm}, 1550 \mathrm{~nm}$ \\
\hline telescope transmission factor & $T_{T}=T_{R}$ & 0.8 \\
\hline pointing loss & $L_{p}$ & 0.3 \\
\hline
\end{tabular}

\section{APPENDIX III \\ DEFAULT PARAMETERS}

For the altitude of the LEO satellite we assume $500 \mathrm{~km}$, which thus represents the lower limit of the link distance. For an elevation angle of, e.g., $15^{\circ}$, the link distance is of some $1400 \mathrm{~km} . .^{13}$

Geostationary satellites have an altitude of $36000 \mathrm{~km}$. Again, the link distance may be larger, depending on the elevation of the satellite (for the ARTEMIS-OGS link the link distance is $41229 \mathrm{~km})$.

The baseline for the ground aperture is $1 \mathrm{~m}$ because this is the telescope diameter of ESA's optical ground station at Tenerife (OGS). Telescopes with a diameter of 20 to $30 \mathrm{~cm}$ are small and light enough to be operated even onboard a small LEO satellite. Larger telescopes are feasible, especially for GEO satellites.

We assume the transmission factors $T_{P}, T_{R}$ of the involved telescopes to be 0.8 . The pointing loss is $L_{P}=0.2$ for all links except for the LEO-LEO link, where we assume $L_{P}=0.3$ to take into account the-possibly - high relative velocity of the satellites that might result in reduced tracking accuracy.

The assumption of an atmospheric attenuation of $A_{a t m}=$ $1 \mathrm{~dB}$ applies for excellent sight conditions (no haze, fog, or clouds) and is valid only in certain wavelength regions.

A recently obtained estimate for the Fried parameter, valid for the optical ground station at Tenerife (OGS), is $r_{0}=90 \mathrm{~mm}$ for a wavelength of $800 \mathrm{~nm}$ in case of weak turbulence [33].

\footnotetext{
${ }^{13}$ Note that for low elevation angles the influence of the atmosphere is increased, a fact not taken into account by the model used here.
}

TABLE V

PARAMETERS FOR LEO-GEO LINKS

\begin{tabular}{l|c|l}
\hline link distance & $L$ & $35500 \mathrm{~km}$ \\
\hline aperture & $D_{T}=D_{R}$ & 20 to $30 \mathrm{~cm}$ \\
\hline wavelength & $\lambda$ & $800 \mathrm{~nm}, 1550 \mathrm{~nm}$ \\
\hline telescope transmission factor & $T_{T}=T_{R}$ & 0.8 \\
\hline pointing loss & $L_{p}$ & 0.2 \\
\hline
\end{tabular}

TABLE VI

PARAMETERS FOR GEO-GEO LINKS

\begin{tabular}{l|c|l}
\hline link distance & $L$ & $40000 \mathrm{~km}$ \\
\hline aperture & $D_{T}=D_{R}$ & 20 to $30 \mathrm{~cm}$ \\
\hline wavelength & $\lambda$ & $800 \mathrm{~nm}, 1550 \mathrm{~nm}$ \\
\hline telescope transmission factor & $T_{T}=T_{R}$ & 0.8 \\
\hline pointing loss & $L_{p}$ & 0.2 \\
\hline
\end{tabular}

For the calculations presented we have assumed wavelengths of $800 \mathrm{~nm}$ and of $1550 \mathrm{~nm}$. Tables II-VI summarize the link characteristics.

\section{ACKNOWLEDGMENT}

We wish to thank J. M. Perdigues Armengol for monitoring and supporting this work.

\section{REFERENCES}

[1] E. Schrödinger, "Die gegenwärtige Situation in der Quantenmechanik," Naturwissenschaften, vol. 23, pp. 807-812, 1935.

[2] A. K. Ekert, "Quantum cryptography based on bell's theorem," Phys. Rev. Lett., vol. 67, pp. 661-663, 1991.

[3] T. Jennewein, C. Simon, G. Weihs, H. Weinfurter, and A. Zeilinger, "Quantum cryptography with entangled photons," Phys. Rev. Lett., vol. 84 , p. $4729,2000$.

[4] W. Tittel, J. Brendel, H. Zbinden, and N. Gisin, "Quantum cryptography using entangled photons in energy-time bell states," Phys. Rev. Lett., vol. 84, p. $4737,2000$.

[5] D. S. Naik, C. G. Peterson, A. G. White, A. J. Berglund, and P. G. Kwiat, "Entangled state quantum cryptography: eavesdropping on the ekert protocol," Phys. Rev. Lett., vol. 84, p. 4733, 2000.

[6] K. Mattle, H. Weinfurter, P. G. Kwiat, and A. Zeilinger, "Dense coding in experimental quantum communication," Phys. Rev. Lett., vol. 76, no. 25, pp. 4656-4659, 1996.

[7] C. H. Bennett, G. Brassard, C. Crépeau, R. Jozsa, A. Peres, and W. K. Wootters, "Teleporting an unknown quantum state via dual classical and Einstein-Podolsky-Rosen channels," Phys. Rev. Lett., vol. 70, no. 13, pp. 1895-1899, 1993.

[8] G. Brassard, Quantum Communication Complexity (A Survey), 2001.

[9] H. Buhrman, W. V. Dam, P. Høyer, and A. Tapp, "Multiparty quantum communication complexity," Phys. Rev. A, vol. 60, pp. 2737-2741, 1999.

[10] C. Brukner, M. Zukowski, and A. Zeilinger, "Quantum communication complexity protocol with two entangled qutrits," Phys. Rev. Lett., vol. 89, p. 197901, 2002.

[11] E. Waks, A. Zeevi, and Y. Yamamoto, "Security of quantum key distribution with entangled photons against individual attacks," Phys. Rev. A, vol. 65, p. 52 310, 2002.

[12] N. Gisin, G. Ribordy, W. Tittel, and H. Zbinden, "Quantum cryptography," Rev. Mod. Phys., vol. 74, pp. 145-195, 2002.

[13] H. Kosaka, A. Tomita, Y. Nambu, T. Kimura, and K. Nakamura, SinglePhoton Interference Experiment Over $100 \mathrm{~km}$ for Quantum Cryptography System Using a Balanced Gated-Mode Photon Detector, 2003.

[14] Z. Yuan, C. Gobby, and A. J. Shields, "Quantum key distribution over $101 \mathrm{~km}$ telecom fiber," in CLEO-Europe, Munich, 2003.

[15] M. Żukowski, A. Zeilinger, M. A. Horne, and A. K. Ekert, “"Eventready-detectors" Bell experiment via entanglement swapping," Phys. Rev. Lett., vol. 71, no. 26, pp. 4287-4290, 1993.

[16] H.-J. Briegel, W. Dür, J. I. Cirac, and P. Zoller, "Quantum repeaters: the role of imperfect local operations in quantum communication," Phys. Rev. Lett., vol. 81, pp. 5932-5935, 1998. 
[17] D. Bouwmeester, J.-W. Pan, K. Mattle, M. Eibl, H. Weinfurter, and A. Zeilinger, "Experimental quantum teleportation," Nature, vol. 390, pp. $575-579,1997$

[18] J.-W. Pan, D. Bouwmeester, H. Weinfurter, and A. Zeilinger, "Experimental entanglement swapping: entangling photons that never interacted," Phys. Rev. Lett., vol. 80, pp. 3891-3894, 1998.

[19] T. Jennewein, G. Weihs, J.-W. Pan, and A. Zeilinger, "Experimental nonlocality proof of quantum teleportation and entanglement swapping," Phys. Rev. Lett., vol. 88, p. 17903, 2001.

[20] J.-W. Pan, S. Gasparoni, R. Ursin, G. Weihs, and A. Zeilinger, "Experimental entanglement purification," Nature, vol. 423, pp. 417-422, 2003.

[21] I. Marcikic, H. de Riedmatten, W. Tittel, H. Zbinden, and N. Gisin, "Long-distance teleportation of qubits at telecommunication wavelengths," Nature, vol. 421, p. 509, 2003.

[22] J.-W. Pan, S. Gasparoni, M. Aspelmeyer, T. Jennewein, and A. Zeilinger, "Freely propagating teleported qubits," Nature, vol. 421, p. 721, 2003.

[23] P. R. Tapster, J. G. Rarity, and P. C. M. Owens, "Violation of bell's inequality over 4 km of optical fiber," Phys. Rev. Lett., vol. 73, p. 1923, 1994.

[24] W. Tittel, J. Brendel, H. Zbinden, and N. Gisin, "Violation of bell inequalities by photons more than $10 \mathrm{~km}$ apart," Phys. Rev. Lett., vol. 81, p. $3563,1998$.

[25] G. Weihs, T. Jennewein, C. Simon, H. Weinfurter, and A. Zeilinger, "Violation of bell's inequality under strict einstein locality condition," Phys. Rev. Lett., vol. 81, p. 5039, 1998.

[26] W. T. Buttler, R. J. Hughes, P. G. Kwiat, S. K. Lamoreaux, G. G. Luther, G. L. Morgan, J. E. Nordholt, C. G. Peterson, and C. M. Simmons, "Practical free-space quantum key distribution over 1 km," Phys. Rev. Lett., vol. 81, pp. 3283-3286, 1998.

[27] R. J. Hughes, J. E. Nordholt, D. Derkacs, and C. G. Peterson, "Practical free-space quantum key distribution over $10 \mathrm{~km}$ in daylight and at night," New Journal of Physics, vol. 4, p. 43, 2002.

[28] C. Kurtsiefer, P. Zarda, M. Halder, H. Weinfurter, P. Gorman, P. Tapster, and J. Rarity, "A step toward global key distribution," Nature, vol. 419, p. $450,2002$.

[29] M. Aspelmeyer, H. R. Böhm, T. Gyatso, T. Jennewein, R. Kaltenbaek, M. Lindenthal, G. Molina-Terriza, A. Poppe, K. Resch, M. Taraba, R. Ursin, P. Walther, and A. Zeilinger, "Long-distance free-space distribution of quantum entanglement," Science, vol. 301, p. 621, June 19, 2003.

[30] H. Horvath, L. A. Arboledas, F. J. Olmo, O. Jovanović, M. Gangl, W. Kaller, C. Sánchez, H. Sauerzopf, and S. Seidl, "Optical characteristics of the aerosol in Spain and Austria and its effect on radiative forcing," J. Geophys. Res., vol. 107(D19), p. 4386, 2002.

[31] C. Bennett, C. Fuchs, and J. Smolin, "Entanglement-enhanced classical communication on a noisy quantum channel," in Quantum Communication, Computing, and Measurement, O. Hirota, A. S. Holevo, and C. M. Caves, Eds. New York: Plenum, 1996, pp. 79-88.

[32] R. C. Ramsey, "Spectral irradiance from stars and planets, above the atmosphere, from 0.1 to 100.0 microns," Appl. Opt., vol. 1, pp. 465-471, 1962.

[33] M. Pfennigbauer and W. R. Leeb, Optical Telescopes for Intersatellite Link-Feasibility Study, ESTEC Contract No. 15872/01, Institut für Nachrichtentechnik und Hochfrequenztechnik, TU Wien, 2002.

[34] D. Bouwmeester, A. Ekert, and A. Zeilinger, Eds., The Physics of Quantum Information. Berlin: Springer-Verlag, 2000.

[35] J. G. Rarity, P. R. Tasper, P. M. Gorman, and P. Knight, "Ground to satellite secure key exchange using quantum cryptography," New J. Physics, no. 4, pp. 82.1-82.21, 2002.

[36] Y.-H. Kim, S. P. Kulik, and Y. Shih, "Quantum teleportation of a polarization state with a complete bell state measurement," Phys. Rev. Lett., vol. 86, pp. 1370-1373, 2001.

[37] M. Zukowski, A. Zeilinger, and H. Weinfurter, "Entangling photons radiated by independent pulsed sources," in Fundamental Problems in Quantum Theory: A Conference Held in Honor of Professor John A. Wheeler, D. M. Greenberger and A. Zeilinger, Eds. New York: NY Acad. Sci., 1995, pp. 91-102.

[38] W. K. Wooters and W. H. Zurek, "A single quantum cannot be cloned," Nature, vol. 299, p. 802, 1982.

[39] R. Kaltenbaek, M. Aspelmeyer, M. Pfennigbauer, T. Jennewein, C. Brukner, W. R. Leeb, and A. Zeilinger, "Proof-of-concept experiments for quantum physics in space," SPIE Proc. Quantum Commun. Quantum Imaging, 2003.

[40] A. Mair, A. Vaziri, G. Weihs, and A. Zeilinger, "Entanglement of the orbital angular momentum states of photons," Nature, vol. 412, p. 313, 2001.
[41] C. Fuchs, N. Gisin, R. B. Griffiths, C. S. Niu, and A. Peres, "Optimal eavesdropping in quantum cryptography. I. Information bound and optimal strategy," Phys. Rev. A, vol. 56, pp. 1163-1172, 1997.

[42] S. Aerts, P. Kwiat, J.-Å. Larsson, and M. Zukowski, "Two-photon franson-type experiments and local realism," Phys. Rev. Lett., vol. 83, p. $2872,1999$.

[43] M. Reyes, Z. Sodnik, P. Lopez, A. Alonso, T. Viera, and G. Oppenhäuser, "Preliminary results of the in-orbit test of ARTEMIS with the optical ground station," in Proc. SPIE, Free-Space Laser Communication Technologies XIV, vol. 4635, Jan. 2002, pp. 38-49.

[44] K. A. Winick, "Atmospheric turbulence-induced signal fades on optical heterodyne communication links," Applied Optics, vol. 25, no. 11, pp. 1817-1825, 1986.

[45] L. C. Andrews and R. L. Phillips, Laser Beam Propagation Through Random Media. Bellingham, WA: SPIE, 1998.

[46] D. L. Fried, "Optical resolution through a randomly inhomogeneous medium for very long and very short exposures," J. Opt. Soc. Amer. vol. 56, pp. 1372-1379, 1966.

[47] G. Gilbert and M. Hamrick, Practical Quantum Cryptography: A Comprehensive Analysis (Part One), 2000.

[48] Z. Sodnik, J. A. Perdigues, and R. H. Czinchy, "Design Data Summary of the ESA Optical Ground Station for In-Orbit Check-Out of Laser Communication Payloads and for the Observation and Registration of Space Debris," ESA/ESTEC, European Space Agency Report, ESA, Ref. no. XA95/267/ZS, 2000

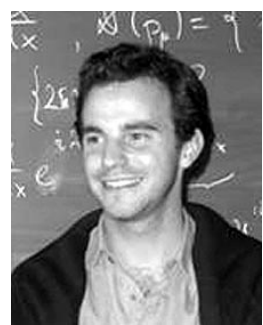

Markus Aspelmeyer was born in Schongau, Germany, in 1974. He studied physics and philosophy at the Ludwig-Maximilians-Universität (LMU), München, Germany, the University of Houston, Houston, Texas, and the Munich School for Philosophy, Munich, Germany. He received the diploma and the Ph.D. degree in experimental physics from LMU in the field of solid-state physics.

In 2002, he was awarded a Feodor-Lynen Fellowship from the Alexander-von-Humboldt Foundation and joined Anton Zeilinger's group at the University of Vienna, Vienna, Austria. His current field of research includes the implementation of long-distance quantum commmunication schemes and fundamental studies on entanglement and multiphoton interference.

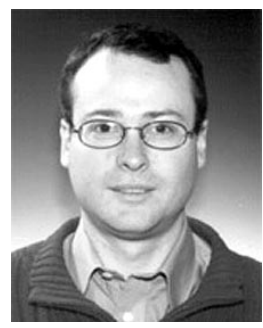

Thomas Jennewein received a degree in experimental physics at the University of Innsbruck, Innsbruck, Austria and the Ph.D. from the University of Vienna, Vienna, Austria. In 2002 he finished his thesis on quantum teleportation and quantum cryptography experiments with entangled photons and was rewarded the "Loschmidt Preis" of the Austrian Chemical-Physical Society for his Ph.D. dissertation in 2002

After one year as a university assistant at the University of Vienna with Prof. Zeilinger and one year in industry, he is now a Senior Researcher at the recently founded Institute for Quantum Optics and Quantum Information, Austrian Academy of Science in Vienna. His research interests are in experiments with entangled photons, such as teleportation of entangled photons, quantum cryptography, the transmission of entangled photons over long distances (including space), quantum interference within optical fibers, testing Bell inequalities, and the development of various electronic devices needed for quantum optics experiments (e.g., photon-detectors, coincidence logics)

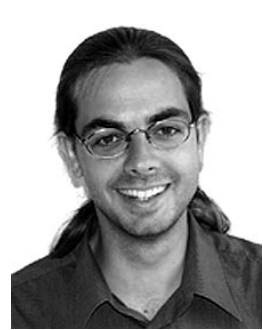

Martin Pfennigbauer (S'00) was born in Tulln, Austria, in November 1973. He received the Dipl.Ing. degree in electrical engineering in 2000 from the Vienna University of Technology, Vienna, Austria, where he is currently pursuing the Ph.D. degree in laser communication systems.

His research interests include direct detection receivers for NRZ and RZ coding and optical intersatellite communications. 


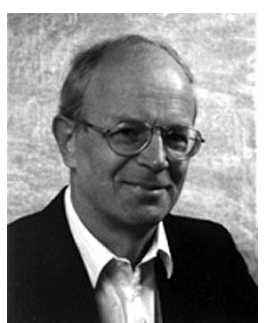

Walter R. Leeb received the MSc. and Dr.Sc.Techn. degrees in electrical engineering from the Technische Universität Wien (TUW), Austria.

He spent two post-doc years at NASA/Goddard Space Flight Center, USA. Later, he obtained the Habilitation for Optical Communications and Laser Techniques. He was a Research Assistant with the Institute of Communications and Radio-Frequency Engineering, TUW, where he became a Lecturer, and has the position of Professor since 1982. He is currently head of the institute. He performed, directed, and managed research and development work in the area of optoelectronic devices (laser modulation, laser frequency control) and of laser communication system aspects (homodyne and heterodyne reception, laser synchronization, optical phase-locked loops, intersatellite laser links, space-borne lidar, optical-phased array antennas, fiber-optic devices, high-data rate photonic wavelength-multiplexed fiber systems). More recently, he also became engaged in stellar interferometry and in quantum communications.

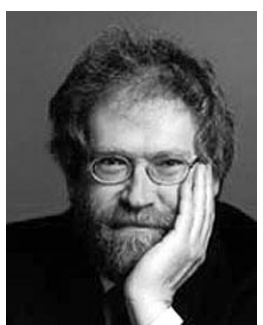

Anton Zeilinger was born in Austria in 1945

He has held positions at universities in Munich, Vienna, Innsbruck, Melbourne, at MIT, and the Collége de France. He is the Director of the Experimental Physics Institute, University of Vienna, Vienna, Austria, and he is Co-Director of the recently founded Institute for Quantum Optics and Quantum Information, Austrian Academy of Science in Vienna, Austria. He and his group have realized in experiment many fundamental predictions of quantum theory and so proved their amazing consequences for our view of the world. These experimental realizations have also laid the foundation for completely new forms of technology, such as fields like quantum cryptography, quantum computation and quantum information processing. His group's major achievements include the world's first quantum teleportation (1997), the first realization of quantum cryptography based on quantum entanglement (1999) and the first demonstration of entanglement purification (2003). His work is to a large extent an application of quantum entanglement, the astonishing feature of quantum physics that Albert Einstein had called "spooky action at a distance."

Prof. Zeilinger has received many awards, among them are an Honorary Professorship at the University of Science and Technology of China, memberships in the German Order Pour le Mérite and various academies, and the Senior Humboldt Fellow Prize of the Alexander von Humboldt-Stiftung. 